\title{
Üç Yüz Yirmibeş Laparoskopik Adrenalektomi Vakasının Retrospektif Analizi: Tek Merkez Deneyimi
}

\author{
Mehmet Çağatay ÇİÇEK ${ }^{1}$, Kadir Ömür GÜNSEREN ${ }^{1}$, Soner CANDER ${ }^{2}$, \\ Hakan VURUŞKAN ${ }^{1}$, İsmet YAVAŞCAOĞLU ${ }^{1}$
}

1 Bursa Uludağ Üniversitesi Tıp Fakültesi, Üroloji Anabilim Dalı, Bursa.

2 Bursa Uludağ Üniversitesi Tıp Fakültesi, Endokrinoloji Bilim Dalı, Bursa.

\begin{abstract}
ÖZET
Kliniğimizde çeşitli adrenal bez patolojileri nedeniyle laparoskopik adrenalektomi (LA) uygulanan hastaların intra-operatif ve post-operatif sonuçlarını değerlendirmeyi amaçladık. 2008 - 2020 yılları arasında LA uygulanan hastaların verileri retrospektif olarak değerlendirildi. Hastaların yaş, cinsiyet gibi demografik özellikleri, adrenal kitlenin boyutu, operasyon süresi, kanama miktarı, preop ve postop hemoglobin değerleri, hastanede kalış süresi, intraoperatif ve postoperatif komplikasyonlar açısından değerlendirildi. Patoloji raporları incelenerek kaydedildi. Çalışmaya 196’sı (\%60.3) kadın ve 129’u erkek olmak üzere toplam 325 hasta dahil edildi. Yüz elliyedi hastaya sağ ve 168 (\%51.7) hastaya sol LA uygulandı. Ortalama yaş $51.6 \pm 12.1$ ve ortalama kitle boyutu $40.3 \pm 20.2 \mathrm{~mm}$ idi. Ortalama operasyon süresi $97 \pm 36.2 \mathrm{dk}$ ve ortalama kanama miktarı $50.8 \pm 44.1 \mathrm{ml}$ idi. Ortalama hastanede kalış süresi $3.1 \pm 2.2$ gün idi. İntraoperatif dönemde bir hastada distal pankreas yaralanması, 2 hastada kanama ile toplam 3 hastada intra-operatif komplikasyon gelişti. Bir hastda açık cerrahiye geçildi. Postoperatif dönemde 7 hastada komplikasyon görüldü. Tüm postoperatif komplikasyonlar ek girişime gerek kalmadan konservatif izlem ile tedavi edildi. LA, adrenal kitlelerin cerrahi tedavisinde tecrübeli merkezlerde güvenle uygulanabilecek efektif ve minimal invazif bir tedavi yöntemdir.
\end{abstract}

Anahtar Kelimeler: Adrenal kitle. Laparoskopik adrenalektomi. Sonuçlar.

Retrospective Analysis of 325 Laparoscopic Adrenalectomy Cases: Single Center Experience

\begin{abstract}
We aimed to evaluate intraoperative and postoperative outcomes of the patients who underwent laparoscopic adrenalectomy (LA) for various adrenal gland pathologies in our clinic. The data of patients who underwent LA between 2008 and 2020 were evaluated retrospectively. The demographic characteristics of the patients such as age and gender, size of the adrenal mass, operation time, estimated blood loss, preoperative and postoperative hemoglobin, length of hospital stay, intraoperative and postoperative complications were evaluated. Pathology reports were examined and recorded. A total of 325 patients, 196 (60.3\%) female and 129 male, were included to study. Right LA was applied to 157 patients and left LA to 168 (51.7\%) patients. The mean age was $51.6 \pm 12.1$ years and the mean tumor size was $40.3 \pm 20.2 \mathrm{~mm}$. The mean operation time was $97 \pm 36.2$ minutes and the mean estimated blood loss was $50.8 \pm 44.1 \mathrm{ml}$. Mean length of hospital stay was $3.1 \pm$ 2.2 days. In the intraoperative period, distal pancreatic injury in one patient, bleeding due to vascular injury in 2 patients, at totally, intraoperative complications developed in 3 patients. In one patient conversion to open surgery was needed. Complications were seen in 7 patients in the post-operative period. All post-operative complications were treated with conservative follow-up without the need for additional intervention. LA is an effective and minimally invasive treatment method that can be safely applied in experienced centers in the surgical treatment of adrenal tumors.
\end{abstract}

Key Words: Adrenal tumor. Laparoscopic adrenalectomy. Outcomes.

Geliş Tarihi: 28.Nisan.2021

Kabul Tarihi: 22.Haziran.2021

Dr. Mehmet Çağatay ÇiçEK

Bursa Uludağ Üniversitesi Tıp Fakültesi

Üroloji Anabilim Dalı

Tel.: 05306452602

E-posta: cgtycicek@gmail.com

Yazarların ORCID ID Bilgisi:

Mehmet Çağatay ÇiÇEK: 0000-0002-0471-5404

Kadir GÜNSEREN: 0000-0001-8673-3093

Soner CANDER: 0000-0001-6303-7896

Hakan VURUSKKAN: 0000-0002-3917-4847

İsmet YAVAŞCAOĞLU: 0000-0002-1788-1997
Cerrahi girişim gerektirecek adrenal patolojiler hastaneye yatan hastalarda 19/100,000 gibi görece seyrek görülmekle birlikte son yıllarda görüntüleme tekniklerindeki ilerlemeler bu patolojilerin daha fazla teşhis ve tedavi edilmesine olanak sağlanmıştır ${ }^{1-4}$. Cerrahi gerektiren adrenal kitleler genellikle hormon aktif kitleler, 4 cm'den büyük non-fonksiyonel adenomlar veya herhangi bir malignitenin adrenal metastazını düşündüren kitleler ve adrenal kaynaklı maligniteler olarak genellenebilir² ${ }^{2}$. Adrenalektomiler açık cerrahi yöntem ve laparoskopik yöntemler kullanılarak uygulanmaktadırlar. Açık operasyon batın duvarında geniş bir 
insizyon gerektirmekte postoperatif dönemde daha yüksek morbidite oranları ve daha uzun hastanede yatış süresine neden olmaktadır ${ }^{5,6}$. Gagner'in 1992 de ilk laparoskopik adrenalektomiyi uygulamasından ${ }^{7} \mathrm{bu}$ yana minimal invaziv tedaviler giderek önem kazanarak günümüzde laparoskopik adrenalektomi (LA) benign adrenal kitlelerin cerrahi tedavisinde altın standart yaklaşım haline gelmiştir ${ }^{2,8-11}$. Adrenal glandın retroperitoneal bölgede olması, sağ tarafta çoğu zaman retrokaval bölgeye uzanması, derin yerleşimli olması ve diğer birçok laparoskopik prosedüre kıyasla daha az sayılarda yapılan bir operasyon olması sebebi ile ileri düzey zorlukta bir laparoskopik operasyon olarak sınıflandırılmaktadır. ${ }^{12}$ Reynolds ve ark. ${ }^{13}$ ise LA öğrenmek için deneyimli bir laparoskopistin az sayıda vaka yapmasının yeterli olduğunu belirtmekle birlikte Rieder ve ark. laparoskopik nefrektomiden aşinalığından dolayı bu yorumun sadece ürologlar için geçerli olabileceğini belirtmişlerdir ${ }^{1}$.

LA için retroperitonoskopik ve transperitoneal yaklaşımlar tarif edilmiştir. Tercih edilecek yöntem cerrahın tecrübesi ve tekniğine göre değişmekle birlikte en sik tercih edilen yöntem transperitoneal anterior yaklaşımdir ${ }^{1}$.

Literatürde yapılan çalışmalar incelendiğinde LA serilerinin sonuçlarının operasyonların yapıldığ 1 sağlık kurumlarının profiline göre, operasyonu uygulayan cerrahın tecrübesine göre ve kurumda yapilan yıllık operasyon sayısına göre değiştiği izlenmektedir ${ }^{9,14}$. Biz bu çalıșmada kliniğimizin 12 yıllık LA deneyimini sunmayı ve literatür eşliğinde intraoperatif ve postoperatif sonuçlarını değerlendirmeyi amaçladık.

\section{Gereç ve Yöntem}

Etik kurul onayının alınmasının (Uludağ Üniversitesi Tıp Fakültesi Etik kurul no:2021-4/29) ardından 2008 - 2020 yılları arasında kliniğimizde adrenal bezde kitle tanısı ile LA uygulanan hastaların verileri retrospektif olarak değerlendirildi. Aynı seansta bilateral LA uygulanan hastalar, preop tetktik ve görüntülemelerinde adrenal bezin primer malignitesi şüphesi olan hastalar, patolojisi adrenal bezin primer malignitesi (adrenokortikal karsinom) olarak raporlanan hastalar, 18 yaşından küçük hastalar çalışmaya alınmadı. Tüm hastalar Üroloji Anabilim Dalı tarafindan transperitoneal lateral LA yöntemiyle opere edildi. Hastaların yaş, cinsiyet gibi demografik özellikleri, adrenal kitlenin boyutu, operasyon süresi, kanama miktarı, preop ve postop hemoglobin değerleri, hastanede kalış süresi, intraoperatif ve postoperatif komplikasyonlar açısından değerlendirildi. Hastaların patoloji raporları incelenerek kaydedildi. Kitle boyutu olarak, patoloji raporlarında ölçülen tümör boyutu esas alındı. Operasyon süresi kamera ve çalışma portlarının yerleştirilmesinden başlanarak cildin kapatılmasına kadar geçen süre ola- rak değerlendirildi. Kanama miktarı ameliyat sırasında aspire edilen ve reseptale biriken sıvı miktarının ölçümü olarak kaydedildi. Preop hemoglobin değeri hastanın ameliyat öncesi yatışında değerlendirilen laboratuar sonucu göre, postop hemoglobin değeri ise ameliyat sonrası birinci gündeki laboratuvar sonucuna göre kaydedildi

Hastaların tamamı ameliyat öncesi bilgisayarlı tomografi veya manyetik rezonans görüntüleme ile değerlendirilmişti. Rutin olarak her hasta için ameliyat öncesi endokrinoloji konsultasyonu istenmiş ve hastaların hormon profilleri değerlendirilmişti. Hastaların ameliyat kararları endokrinoloji kliniği ortak yapılan konseylerde verildi. Postoperatif dönemde hastalar yine endokrinoloji tarafindan değerlendirilerek önerileri uygulandı. Hastaların taburculuk sonrası takipleri yine üroloji ve endokrinoloji bölümleri tarafindan ortak olarak yapıldı.

\section{Bulgular}

Hastaların demografik özellikleri, intraoperatif sonuçları ve komplikasyonlar Tablo I'de sunulmuştur. Çalışmaya 345 hasta dahil edilmesinin ardından dişlama kriterlerinden sonra kalan 325 hastanın 196's1 (\%60.3) kadın ve 129'u erkek idi. 157 hastaya sağ ve 168 (\%51.7) hastaya sol LA uyguland. Hastaların ortalama yaşı $51.6 \pm 12.1$ ve ortalama kitle boyutu $40.3 \pm$ $20.2 \mathrm{~mm}$ idi. Ortalama operasyon süresi $97 \pm 36.2 \mathrm{dk}$ ve ortalama kanama miktarı $50.8 \pm 44.1 \mathrm{ml}$ olarak hesaplandı. Hastaların ortalama preoperatif hemoglobin değeri $12.9 \pm 1.6 \mathrm{~g} / \mathrm{dL}$ iken ortalama postoperatif hemoglobin değerleri $12.1 \pm 5.9 \mathrm{~g} / \mathrm{dL}$ idi. Ortalama hastanede kalış süresinin $3.1 \pm 2.2$ gün olduğu belirlendi. İntraoperatif dönemde bir hastada distal pankreas yaralanması gelişti, iki hastada peroperatif kan transfüzyonu gerektirecek kanama ve bir hastada kanama nedeniyle açığa geçilmesi ile toplam 4 hastada intra-operatif komplikasyon gelişti. Postoperatif komplikasyon; operasyon tarihinden sonraki 30 gün içinde gelişen komplikasyonlar olarak tanımlandı. Postoperatif dönemde 7 hastada komplikasyon görüldü. Komplikasyon gelişen hastalardan dördününde Clavien-Dindo sinıf (CDC) 1 (hafif derecede solunum sıkıntısı), bir hastada CDC 2 (transfüzyon gerektiren kanama), bir hastada CDC 3 (distal pankreas yaralanmasına bağlı pankreatik drenaj) ve bir hastada CDC 4 (intrakranyal hemoraji) komplikasyon gelişti. Hastaların demografik verileri, operatif veriler ve postoperatif komplikasyonlar Tablo I'de özetlemiştir. Tüm postoperatif komplikasyonlar ek girişime gerek kalmadan konservatif izlem ile tedavi edildi. Hastaların patoloji sonuçları Tablo II'de özetlenmiştir. 
Tablo I. Hastaların demografik özellikleri, intraoperatif sonuçlar ve komplikasyonlar.

\begin{tabular}{|c|c|}
\hline Cinsiyet (Kadın/Erkek) (n) & $196 / 129$ \\
\hline Yaş (yıl) & \begin{tabular}{|l|}
$51.6 \pm 12.1$ \\
$52(21-88)$
\end{tabular} \\
\hline Taraf (Sağ/Sol) (n) & $157 / 168$ \\
\hline Boyut (mm) & $\begin{array}{l}40.3 \pm 20.2 \\
36(10-150)\end{array}$ \\
\hline Operasyon Süresi (dk) & $\begin{array}{l}97.0 \pm 36.2 \\
90(20-210)\end{array}$ \\
\hline Kanama Miktarı (ml) & $\begin{array}{l}50.8 \pm 44.1 \\
50(10-500)\end{array}$ \\
\hline Hemoglobin (Preop/Postop) (mg/dL) & $12.9 \pm 1.6 / 12.1 \pm 5.9$ \\
\hline $\begin{array}{r}\text { Hastanede kalış süresi (gün) } \\
\text { (ortalama, SD) } \\
\text { (medyan, min-max) }\end{array}$ & $\begin{array}{l}3.1 \pm 2.2 \\
3(1-26)\end{array}$ \\
\hline \multicolumn{2}{|l|}{ Postoperatif Komplikasyonlar (n) } \\
\hline $\begin{array}{l}\text { Uzamış drenaj (Distal pankreas } \\
\text { yaralanmasına sekonder) }\end{array}$ & $1(\mathrm{CDC} 3)$ \\
\hline Transfüzyon gerektiren kanama & $1(\mathrm{CDC} 2)$ \\
\hline Solunum sıkntısı (Hafif derecede) & 4 (CDC 1) \\
\hline İntrakranial kanama & $1(\mathrm{CDC} 4)$ \\
\hline
\end{tabular}

$\mathrm{n}$ : say1, mm: milimetre, dk: dakika, ml: mililitre, dL: desilitre, SD: standart sapma, min: minimum, max: maksimum, CDC: Clavien- Dindo komplikasyon sinıflaması

Tablo II. Hastaların patoloji sonuçlarına göre dağılımı

\begin{tabular}{|l|l|}
\hline Adenom & 159 \\
\hline Diğer Benign Patolojiler & 60 \\
\hline Feokromasitoma & 57 \\
\hline Malign Feokromasitoma & 1 \\
\hline Metastaz & 48 \\
\hline
\end{tabular}

\section{Tartışma ve Sonuç}

Literatürde LA konusunda yapılan çalışmaların sonuçları değerlendirildiğinde LA, açık cerrahiye oranla daha düşük mortalite ve morbidite oranlarına sahip olması ve ayrıca daha az post operatif ağrı, daha kısa hastanede kalış süresi ve daha iyi kozmetik sonuçlar gibi çeşitli avantajlar sağlamasının yanı sıra. üzerinde bir hayli tartışma olmakla birlikte LA'nın adrenokortikal karsinom tedavisinde dahi açık adrenalektomi ile benzer onkolojik sonuçları olduğunu bildiren çalışmalar mevcuttur ${ }^{1,4,14-19}$. Kliniğimizin LA serisi Avrupa ve Birleşik Devletler'deki merkezlerle kyyaslanabilir sayıda olmasından dolayı bu çalışmada serimizi analiz ederek literatür ile karşılaştırmayı ve bulgularımızı paylaşmayı planladık.

Laparoskopik adrenalektomi retroperitoneal veya transperitoneal yaklaşım ile uygulanabilir ${ }^{1}$. Her iki yaklaşımın çeşitli avantaj ve dezavantajları bulunmaktadır. Retroperitoneal yaklaşım daha önce abdominal cerrahi öyküsü olan hastada batın içi yapışılıklardan uzak kalınmasına olanak sağlarken, daha dar çalışma alanı sunması nedeniyle özellikle büyük adrenal kitlelerin laparoskopik cerrahisinde zorluk oluşturabilir ${ }^{19}$. Suzuki ve ark retroperitoneal yaklaşımı, $5 \mathrm{~cm}$ 'den küçük adrenal kitlelerde ve yeterli cerrahi deneyime sahip olunması halinde önermektedir ${ }^{20}$. Bununla birlikte transperitoreal yaklaşımda karaciğer, pankreas ve dalak gibi adrenal beze komşu intraabdominal organların ekartasyonu gerekmektedir ve bu yaklaşım daha geniş çalışma alanı sağlayarak büyük boyutlu adrenal kitlelerin laparoskopik cerrahisini mümkün k1lar ${ }^{19}$. Biz kliniğimizde geniş laparoskopik böbrek cerrahisi deneyimimizden kaynaklanan tecrübemizden dolayı rutin olarak transperitoneal lateral yaklaşımı tercih etmekteyiz.

Adrenal cerrahilerde önemli bir konu da adrenal kitlenin boyutudur. $\mathrm{Bu}$ parametrenin önemi hem büyük kitlelerin cerrahi diseksiyonu ve eksizyonundaki zorluklardan, hem de kitle boyutunun artması ile malignite riskinin doğru orantılı şekilde yükselmesinden kaynaklanmaktadır, Alemanno ve ark. yayınladığı derlemede 4 cm'den küçük adrenal kitlelerde malignite riski \%2'nin altında iken bu oran $6 \mathrm{~cm}$ 'nin üzerinde $\% 25$ olarak belirtilmiştir ${ }^{21}$. Günümüzde büyük boyutlu kitlelerde LA'nin güvenle uygulanabileceğini bildiren yayınlar bulunmaktadır ${ }^{18,22}$. Biz çalışmamızda ortalama kitle boyutu $40.3 \pm 20.2 \mathrm{~mm}$ ve medyan 36 (10-150) mm olarak saptadık.

Peroperatif kanama, LA'da en sık görülen komplikasyonlardan olmakla birlikte açık cerrahiye geçişi gerektirebilmektedir ${ }^{23}$. Farklı çalışmalarda farklı açığa geçiş oranları bildirilmekle birlikte genel olarak açık cerrahiye geçiş oranları $\% 0.2$ ile $\% 6.2$ arasında değişmektedir $^{24}$. Bizim çalışmamızda sadece bir hastada $(\% 0.3)$ açık cerrahiye geçilmiş̧tir ve oransal olarak literatürdeki bildirilen çalışmalar ile uyum göstermektedir. Laparoskopik adrenaleketomi uygulanan hastalarda transfüzyon gerektirecek kanama \%0 ila \%13.5 arasında değişmektedir ${ }^{25}$. Kanama nedeni daha çok adrenal bezin etraf dokulardan diseksiyonu veya sürrenal venin diseksiyonu ve kapatılıp ligasyonu sırasında ortaya çıkabilmektedir. Carter ve ark.'nın ve bizim önceki çalışmamızda belirtildiğimiz gibi $6 \mathrm{~cm}$ 'den büyük kitlesi olan hastaların daha küçük kitlesi olanlara kıyasla daha fazla kanadığını gösteren çalışmalar olmakla birlikte ${ }^{18}$ bizim serimizdeki kanama miktarı ortalama $50.8 \pm 44.1 \mathrm{ml}$ olup geniş hasta serileri olan çalışmalar ile uyumludur. Açık cerrahiye geçiş ve kanama miktarımızın düşük olmasını kliniğimizin gerek LA, gerekse laparoskopik böbrek cerrahisindeki yüksek tecrübemize bağlı olduğunu düşünmekteyiz.

Operasyon zamanı ele alındığında, literatürde operasyon süresini etkileyen faktörlerin ne olduğu üzerine bir fikir birliği yoktur. Bazı çalışmalar $6 \mathrm{~cm}$ 'den büyük kitlelerde operasyon süresinin daha uzun olduğunu belirtmekte iken, bazı çalışmalar ise kitle boyutu- 
nun operasyon süresini etkilemediği sonucuna varmış$\operatorname{tır}^{26,27}$. Tiberio ve ark. çalışmasında ise multivariate analizde $140 \mathrm{dk}$ 'yı aşan operasyon süresinin perioperatif komplikasyonlarla ilişkili olduğunu saptamışlar$\mathrm{d}_{1} \mathrm{r}^{28}$. Bizim serimizde operasyon süremiz literatür ile benzer olarak ortalama $97 \pm 36.2$ dk, medyan 90 (20210) dk olarak hesaplanmıştır.

Laparoskopik cerrahiler açık cerrahilere göre hastanede kalış süresi açısından daha avantajlıdır ${ }^{29}$. Güncel bir makalede $4 \mathrm{~cm}$ 'den daha büyük adrenal kitleler için yapılan LA'nın hastanede kalış süresini uzattığg, yine benzer olarak başka bir çalışma ise $5 \mathrm{~cm}$ üzerindeki kitleler için aynı sonucu belirtilmiștir ${ }^{30,31}$. Bu sonuçlardan farklı olarak Carter ve ark. Yaptıkları çalışmada $6 \mathrm{~cm}$ 'den küçük ve $6 \mathrm{~cm}$ 'den büyük adrenal feokromasitomalarda hastanede kalıș süresi açısından fark saptamamışlardır ${ }^{32}$. Literatürde Carter ve ark. Çalışması ile benzer sonuçlar bildiren çalışmalar da mevcuttur ${ }^{26,33,34}$. Hastanede kalış süresi ile ilişkilendirilen başka bir faktör de hastaların ASA skorlarıdır. Chen ve ark. ASA skorunun $\geq 3$ olmasının komplikasyon gelişimi ve hastanede kalış süresini uzatan bağımsız bir faktör olduğunu bildirmişlerdir. Biz çalışmamızda literatür ile uyumlu olarak hastanede kalış süresini ortalama $3.1 \pm 2.2$ gün olarak hesapladık.

Sonuç olarak LA, adrenal adenokarsinom cerrahisindeki yeri tartışmalı olmakla birlikte, bunun dişındaki adrenal kitlelerin cerrahi tedavisinde özellikle tecrübeli merkezlerde güvenle uygulanabilecek efektif ve minimal invazif bir cerrahi yöntemdir.

\section{Etik Kurul Onay Bilgisi:}

Onaylayan Kurul: Uludağ Üniversitesi Tıp Fakültesi Klinik Araştırmalar Etik Kurulu

Onay Tarihi: 24.02 .2021

Karar No: 2021-04/29

Araștırmacı Katkı Beyanı:

Fikir ve tasarım; İ.Y., H.V, M.Ç.Ç.; Veri toplama ve işleme M.C..Ç., K.Ö.G.; Analiz ve verilerin yorumlanması S.C., M.Ç.Ç.; Makalenin önemli bölümlerinin yazılması M.Ç.Ç., K.Ö.G., S.C.

Çıkar Çatış̧ması Beyanı:

Makale yazarının çıkar çatışması beyanı yoktur.

\section{Kaynaklar}

1. Rieder JM, Nisbet AA, Wuerstle MC, Tran VQ, Kwon EO, Chien GW. Differences in left and right laparoscopic adrenalectomy. JSLS 2010;14(3):369-73.

2. Fiszer P, Toutounchi S, Pogorzelski R, Krajewska E, Cieśla W, Skórski M. Laparoscopic adrenalectomy--assessing the learning curve. Pol Przegl Chir 2012;84(6):293-7.

3. Gupta PK, Natarajan B, Pallati PK, et al.: Outcomes after laparoscopic adrenalectomy. Surg endosc 2011; 25(3): 784-94.

4. Brix D, Allolio B, Fenske W, et al. Laparoscopic versus open adrenalectomy for adrenocortical carcinoma: surgical and oncologic outcome in 152 patients, Eur. Urol 2010;58:609-15.

5. Wang HS, Li CC, Chou YH, Wang CJ, Wu WJ, Huang CH Comparison of laparoscopic adrenalectomy with open surgery for adrenal tumors. Kaohsiung J Med Sci 2009;25:438-44.
6. Vargas HI, Kavoussi LR, Bartlett DL, et al. Laparoscopic adrenalectomy: A new standard of care. Urology 1997;49:67378.

7. Gagner M, Lacroix A, Bolte' E. Laparoscopic adrenalectomy in Cushing's syndrome and pheochromocytoma. N Engl J Med 1992;327:1033.

8. Smith CD, Weber CJ, Amerson JR. Laparoscopic adrenalectomy: new gold standard. World J Surg 1999 Apr;23(4):389-96.

9. Gumbs AA, Gagner M. Laparoscopic adrenalectomy. Best Pract Res Clin Endocrinol Metab 2006 Sep;20(3):483-99.

10. Agrusa A, Romano G, Frazzetta G, et al. Laparoscopic adrenalectomy for large adrenal masses: single team experience. Int $\mathrm{J}$ Surg 2014;12 Suppl 1:S72-4.

11. Erbil Y, Barbaros U, Karaman G, Bozbora A, Ozarmağan S. The change in the principle of performing laparoscopic adrenalectomy from small to large masses. Int J Surg 2009 Jun;7(3):266-71.

12. Maccabee DL, Jones A, Domreis J, Deveney CW, Sheppard BC. Transition from open to laparoscopic adrenalectomy: the need for training. Surg Endosc 2003;10:1566-9.

13. Reynolds FD, Goudas L, Zuckerman RS, Gold MS, Heneghan S. A rural community based program can train surgical residents in advanced laparoscopy. J Am Coll Surg 2003;4:620-3.

14. Pędziwiatr M, Wierdak M, Ostachowski M, et al. Single center outcomes of laparoscopic transperitoneal lateral adrenalectomy-Lessons learned after 500 cases: A retrospective cohort study. Int J Surg. 2015 Aug;20:88-94.

15. Murphy MM, Witkowski ER, Ng SC, et al. Trends in adrenalectomy: a recent national review. Surg Endosc 2010;24:251826.

16. Miller BS, Gauger PG, Hammer GD, Doherty GM. Resection of adrenocortical carcinoma is less complete and local recurrence occurs sooner and more often after laparoscopic adrenalectomy than after open adrenalectomy. Surgery 2012;152:1150-7.

17. Cooper AB, Habra MA, Grubbs EG, et al. Does laparoscopic adrenalectomy jeopardize oncologic outcomes for patients with adrenocortical carcinoma? Surg Endosc 2013;27:4026-32.

18. Cicek MC, Gunseren KO, Senol K, Vuruskan H, Yavascaoglu I. Is $6 \mathrm{~cm}$ Diameter an Upper Limit for Adrenal Tumors to Perform Laparoscopic Adrenalectomy? J Laparoendosc Adv Surg Tech A 2021;31(3):301-5.

19. Sautter AE, Cunningham SC, Kowdley GC. Laparoscopic Adrenalectomy for Adrenal Cancer-A Systematic Review. Am Surg 2016;82(5):420-6.

20. Suzuki K, Kageyama S, Hirano Y, et al. Comparison of 3 surgical approaches to laparoscopic adrenalectomy: a nonrandomized, background matched analysis. J Urol 2001;166:437_ 43.

21. Alemanno G, Bergamini C, Prosperi P, Valeri A. Adrenalectomy: indications and options for treatment. Updates Surg 2017;69(2):119-25

22. Bozkurt IH, Arslan M, Yonguc T, et al. Laparoscopic adrenalectomy for large adrenal masses: Is it really more complicated? Kaohsiung J Med Sci. 2015 Dec;31(12):644-8.

23. Di Buono G, Buscemi S, Lo Monte AI, et al. Laparoscopic adrenalectomy: preoperative data, surgical technique and clinical outcomes. BMC Surg 2019;18:128.

24. Nguyen PH, Keller JE, Novitsky YW, Heniford BT, Kercher KW. Laparoscopic approach to adrenalectomy: Review of perioperative outcomes in a Single Center. Am Surg 2011;77:5926.

25. Conzo G, Pasquali D, Della Pietra D, et al. Laparoscopic adrenal surgery: ten-year experience in a single institution. BMC Surg 2013;13 suppl2:S5.

26. Balla A, Palmieri L, Meoli F, et al. Are adrenal lesions of $6 \mathrm{~cm}$ or more in diameter a contraindication to laparoscopic adrenalectomy? A case control study. World J Surg 2020;44:810-8. 


\section{Laparoskopik Adrenalektomi}

27. Pearlstein SS, Kuo JH, Chabot JA, Lee JA. Periadrenal volume is a better predictor of prolonged operative time in laparoscopic retroperitoneal adrenalectomy than BMI. World J Surg 2020;44:578-84.

28. Tiberio GAM, Solaini L, Arru L, Merigo G, Baiocchi GL, Giulini SM. Factors influencing outcomes in laparoscopic adrenal surgery. Langenbecks Arch Surg 2013;398:735-43.

29. Zhu W, Wang S, Du G, Liu H, Lu J, Yang W. Comparison of retroperitoneal laparoscopic versus open adrenalectomy for large pheochromocytoma: a single-center retrospective study. World J Surg Oncol. 2019;17(1):111.

30. Chen Y, Scholten A, Chomsky-Higgins K, et al. Risk factors associated with perioperative complications and prolonged length of stay after laparoscopic adrenalectomy. JAMA Surg 2018;153:1036-41.
31. Chen W, Liang Y, Lin W, Fu GQ, Ma ZW. Surgical management of large adrenal tumors: Impact of different laparoscopic approaches and resection methods on perioperative and longterm outcomes. BMC Urol 2018;18:31.

32. Carter YM, Mazeh H, Sippel RS, Chen H. Safety and feasibility of laparoscopic resection for large ( $\neq 6 \mathrm{CM}$ ) pheochromocytomas without suspected malignancy. Endocr Pract 2012;18:7206.

33. Chung HS, Kim MS, Yu HS, et al. Laparoscopic adrenalectomy using the lateral retroperitoneal approach: Is it a safe and feasible treatment option for pheochromocytomas larger than 6 cm? Int J Urol 2018;25:414-9.

34. Hwang I, Jung SI, Yu SH, Hwang EC, Yu HS, Kim SO. Is larger tumor size a contraindication to retroperitoneal laparoscopic adrenalectomy? World J Urol 2014;32:723-8. 
